\title{
Childhood white matter disorders: much more than just diseases of myelin
}

\author{
Marianna Bugiani $^{1}\left[{ }^{[} \cdot\right.$ Marjo S. van der Knaap $^{1}$
}

Published online: 18 July 2017

(C) Springer-Verlag GmbH Germany 2017

Childhood white matter disorders and their sequelae constitute a major clinical problem associated with considerable morbidity and mortality. The genetic forms occur in at least 1:7500 live births, while the overall incidence of acquired white matter afflictions is not known, comprising amongst others congenital infections, perinatal damage, and inflammatory disorders. The emotional and economic burdens for patients, families, and society are enormous. Understanding the pathophysiology of childhood white matter disorders is thus necessary to find new openings for treatment and, if possible, cure. This issue of Acta Neuropathologica presents a two-piece mini-cluster on white matter disorders in children, focussing on their neuropathology and disease mechanisms.

The brain white matter is made up of axons and ensheathing myelin, oligodendrocytes, astrocytes, blood vessels, microglia, and other non-neural cells, all embedded in an extracellular matrix. It develops in utero and during the first years of post-natal life, by mechanisms of cell specification, differentiation, and maturation driven by intrinsic cues and regulated by inter-cellular interactions. A central feature of white matter development is deposition of myelin around receptive axons. This is carried out by oligodendrocytes once they have reached full maturity. Perturbation of oligodendrocyte maturation is, therefore, a mechanism that can interfere with developmental myelination and myelin regeneration upon injury.

The first article of the mini-cluster describes the neuropathology and pathophysiology of white matter injury in the preterm infant. Due to major improvements in

Marianna Bugiani

m.bugiani@vumc.nl

1 VU Medisch Centrum, Amsterdam, The Netherlands the quality of assistance to the preterm child, white matter injury with limited necrosis has largely replaced the cystic necrotic periventricular leukomalacia. In his exhaustive piece, Back describes the selective maturationdependant vulnerability of late oligodendrocyte progenitors (pre-oligodendrocytes) to oxidative stress induced by hypoxia-ischemia or maternal-foetal infection. Pre-oligodendrocytes are pre-myelinating cells still able to divide, but unable to migrate. They abundantly populate the brain white matter between 23 and 32 week post-conception, the high-risk period for developing white matter injury. At earlier and later post-conceptional ages, oligodendrocytes are present in different developmental stages that make them more resistant to intrinsic and extrinsic sources of oxidative stress. This damage ultimately perturbs the normal developmental myelination as a result of the death of pre-oligodendrocytes with consequent scarcity of their more mature myelinating forms.

A concept emerging in white matter biology and disease is that integrity and proper function of the white matter are closely related to all its structural constituents. This concept is supported by increasing knowledge on white matter biology and identification of genetic white matter disorders due to defects in proteins expressed by cells other than the oligodendrocytes. In the second article of this mini-cluster, van der Knaap and Bugiani highlight how these achievements have changed the view on the pathophysiology of genetic white matter disorders, the leukodystrophies. Once considered to exclusively be diseases of myelin, either directly or through the oligodendrocytes, they are now acknowledged as diseases deriving from defects in any of the white matter structural components, including oligodendrocytes, astrocytes, microglia, axons, and blood vessels. The authors offer an overview of the disease mechanisms underlying numerous disorders and put forward a 
challenging novel cellular pathology-based classification that distinguishes oligodendrocytopathies from astrocytopathies, microgliopathies, leuko-axonopathies, and leuko-vasculopathies.

To the careful reader, the two articles of the mini-cluster have points in common. The most intriguing is that similar pathomechanisms may occur in acquired and genetic white matter disorders, opening the possibility of shared reparative therapies. In white matter injury, the immature white matter reacts to the death of pre-oligodendrocytes by increasing the rate of cell proliferation. This proliferative response expands the pool of early progenitor cells which, however, fail to differentiate beyond the pre-oligodendrocyte stage. One signalling pathway involved in disrupted myelination involves hyaluronan, a glycosaminoglycan and major component of the extracellular matrix. Hyaluronan is secreted by astrocytes and also regulates the maturation of pre-oligodendrocytes into myelinating cells. The same mechanism occurs in the genetic astrocytopathy vanishing white matter disease, where profoundly dysfunctional astrocytes overproduce hyaluronan. In vanishing white matter disease as in white matter injury of the preterm, this results in an arrest of the maturation of pre-oligodendrocytes into mature, myelin-forming cells, and ultimately in a profound failure of the white matter intrinsic regenerative response to injury. This highlights the major message of the mini-cluster, which is that understanding the multifaceted nature of white matter biology and pathophysiology is the prerequisite for elaborating strategies for repair. 